\title{
PAPER
}

\section{Predicting the outcome of cholinesterase inhibitor treatment in Alzheimer's disease}

\section{P J Connelly, N P Prentice, K G Fowler}

See Editorial Commentary, p 305

See end of article for authors' affiliations ......................

Correspondence to: P J Connelly, Consultant Old Age Psychiatrist, Honorary Senior Lecturer, Department of Psychiatry, University of Dundee, Murray Royal Hospital Muirhall Road, Perth, $\mathrm{PH} 2$ 7BH, UK; pic@dementiaresearch. freeserve.co.uk

Received 16 April 2004 In revised form 8 November 2004 Accepted

9 November 2004
Objective: To investigate the possibility that response to cholinesterase inhibitor therapy could be predicted by easily measurable variables that are known to change as a result of treatment (such as the Mini Mental State Examination), measures of function (such as the instrumental activities of daily living and the social behaviour subscales of the Nurse's Observation Scale for Geriatric Patients), and measures of attention (such as the Digit Symbol Substitution Test; DSST), or that might influence response through structural (for example, age, cerebrovascular disease, medial temporal lobe (MTL) atrophy, hypertension) or chemical (for example, smoking) mechanisms.

Method: This was a cohort study of 160 consecutive outpatients with probable Alzheimer's disease who commenced cholinesterase inhibitor treatment over a 3 year period in a semi-rural area of Scotland. Results: The overall response rate was $42.1 \%$. Stratification of response between good and poor responders was possible using baseline DSST and a measure of MTL thickness using CT. Among the patients, $60.4 \%$ of those above the cut off point for both DSST and MTL thickness (29/48 subjects) were classified as good responders, compared with $6.3 \%$ of subjects below the cut off point for both (1/16 subjects). Subjects above the cut off point for both measures were more likely to be classified as good responders than subjects with only one or no values above the respective cut off points $\left(\chi^{2}=10.61, d f=1\right.$, $\mathrm{p}=0.001$ )

Conclusions: The DSST and a measure of MTL thickness derived from CT scanning may be useful in improving the prediction of response to cholinesterase inhibitors in subjects with AD. Subjects with low DSST scores and more severe MTL atrophy are unlikely to respond to treatment. These preliminary data justify a prospective trial of the usefulness of our suggested predictive measures.
V riables that might predict the outcome of cholinesterase inhibitors include measurements shown to change in double blind randomised placebo controlled trials including cognition, ${ }^{1-3}$ levels of functioning, ${ }^{23}$ and behaviour. ${ }^{3}$ Parameters that might affect response through possible structural mechanisms, (such as age, level of hippocampal atrophy and vascular change), or chemical mechanisms (such as smoking), could also help to discriminate responders and non-responders.

Baseline measures, such as severity of cognitive impairment, ${ }^{4}$ rate of pre-treatment decline, ${ }^{5}$ older age, ${ }^{6}$ current smoking, ${ }^{7}$ and the presence of concurrent vascular risk factors, ${ }^{8}$ have been reported as influencing response in separate studies. The role of the E4 allele of apolipoprotein $\mathrm{E}$ is controversial. Impaired response, ${ }^{9}$ no effect, ${ }^{10}$ and improved response $^{11}$ have all been associated with its presence. Hippocampal volume was associated with response to a single dose of tacrine in one study, ${ }^{12}$ but not in a larger study with stricter outcome criteria by the same group. ${ }^{13}$ An inverse relationship between atrophy of the substantia innominata and response to 6 months' treatment with donepezil has been described, ${ }^{14}$ although only the Mini Mental State Examination (MMSE) $)^{15}$ was used as an outcome measure.

Improvement in attentional rather than memory measures following chronic tacrine treatment has been reported, ${ }^{16}$ but the authors did not relate measures of baseline performance to ultimate outcome. Improvement in attention after single doses of tacrine ${ }^{12} 131718$ did not correlate with response over a 52 week period. ${ }^{18}$ An association between reduction in theta power following cholinesterase inhibitor administration and cognitive outcome has been demonstrated in two small studies. ${ }^{19} 20$
Cholinesterase inhibitors are expensive and have significant side effects. Patients, clinicians, and managers would benefit if these drugs could be targeted at those most likely to respond in a clinical setting. Our service in Perth and Kinross is in a large semi-rural area in central Scotland with no specialised memory clinic. The service has a local CT scanner but very restricted access to MRI or EEG.

In this cohort study of subjects $>45$ years with NINCDSADRDA probable Alzheimer's disease (AD), ${ }^{21}$ who were treated with cholinesterase inhibitor treatment during a 3 year period, we wished to establish whether any baseline variable or combination of variables could discriminate eventual "good" and "poor" responders after 6 months of treatment, using inexpensive, rapid, and effective tests, including neuroimaging. Additionally, while recognising the possible aetiological role of vascular changes in $\mathrm{AD}$, we wished to examine the effect of vascular risk factors, such as hypertension and smoking, and the presence of vascular change on CT scan.

\section{MATERIALS AND METHODS}

Consecutive subjects with probable AD who scored 11-26 on the MMSE and $<2$ on the Rosen scale, ${ }^{22}$ with no evidence of significant cerebrovascular disease (see below) were commenced on either donepezil, rivastigmine, or galantamine. There were no restrictions on the choice of drug. Subjects with focal neurological (long tract) signs and symptoms were

Abbreviations: $A D$, Alzheimer's disease; DSST, Digit Symbol Substitution Test; IADL, instrumental activities of daily living; MMSE, Mini Mental State Examination; MoM, multiple of the median; MTL, medial temporal lobe; NOSGER, Nurse's Observation Scale for Geriatric Patients; SB, social behaviour 
excluded. Subjects with concurrent functional psychosis, alcohol related dementia, head injury, infective brain disease, a clear history of systemic metabolic upset, or major physical problems that contraindicated cholinesterase inhibitor treatment were also excluded. Stable physical problems were accepted, as were subjects who lived alone if they had regular visits from a carer or if they were known to be compliant with other medication. Smoking and alcohol histories were recorded. Data were entered prospectively into a dedicated spreadsheet.

Vascular change on CT was assessed by considering distribution and severity of peri-ventricular white matter change, including infarcts. We defined significant cerebrovascular disease as vascular change on CT in subjects with marked gait disturbance, frequent falls, urinary frequency or urgency, ${ }^{23}$ and poor performance of Luria motor tests ${ }^{24-26}$ and tests of frontal lobe function. These subjects were excluded from treatment. Subjects with radiological vascular changes in the absence of these symptoms and signs were included.

At baseline, cognition was assessed using the MMSE, ${ }^{16}$ Kew cognitive map, ${ }^{27}$ Digit Symbol Substitution Test (DSST), ${ }^{28}$ verbal fluency, finger tap, bimanual reciprocal coordination, and motor sequence. ${ }^{29}$ Functional ability was assessed by the Nurse's Observation Scale for Geriatric Patients (NOSGER), ${ }^{30}$ completed by the carer. Each item on the subscales was scored 1 ("never") to 5 ("all the time"), except when the reverse was indicated by the scale's authors.

\section{Medial temporal lobe (MTL) measurement}

CT scans were undertaken using a Toshiba XvisionGX scanner prior to February 2001 and a GE Hispeed NXI thereafter. Measures of intra-cerebral structures were performed on screen during analysis of the scan using electronic callipers. Standard axial views were augmented by temporal lobe orientated slices similar to the technique described by Jobst et al. ${ }^{31}$ Allowing for patient position and technical limitations, this equates to scanning 25-30 degrees reverse angle to the orbitomeatal line, or 15-20 degrees reverse angle to the anthropological line, which approximates to a line from the inner tip of the nasal cartilage to the pituitary fossa. As recommended, $2 \mathrm{~mm}$ slices were performed in this orientation, avoiding the orbits. "Minimal thickness" measured the hippocampus directly from the hippocampal fissure to the medial wall of the temporal lobe, avoiding the choroid plexus. The lesser of the left or right minimum values was then converted to the multiple of the median (MoM) age related value, using the formula of Jobst et al, ${ }^{31}$ to control for the influence of age on the absolute value of the measure. This is a well validated measure that we felt would allow quantitative assessment of atrophy, and be more accurate than semi-qualitative measures in determining the influence of atrophy on response to treatment. We termed this variable MoMl.

From the same series of slices, a further measurement of the full thickness of the MTL was made across the parahippocampal gyrus and white matter arising directly from the hippocampus, which avoided sulcal and partial volume effects - that is, a measurement of the medial part of the temporal lobe from the medial wall of the lateral ventricle to the ambient cistern, avoiding the choroid plexus. Empirically, we converted the value of the average of the right and left thickness obtained by this measurement to a multiple of the median using the same formula and values for age related norms as in the calculation of MoMl. We believe that taking an average rating reduces adverse influences such as rotation of the subject's head during the scanning procedure. This variable was termed MoM2. Although a learning curve exists for radiologists and radiographers, adequate views and consistency of measurement can be obtained after about 20-25 scans.

All measurements were undertaken by a single radiologist experienced in the procedure, who was blinded to the subject's diagnosis and severity of dementia. Intra-rater reliability of the MoM2 measurement was assessed by reevaluation of 25 scans-that is, 50 measurements. Intra-class correlation was $0.8(\mathrm{p}<0.0005)$.

\section{Dose titration}

Standard titration protocols were used during the first 3 months. All subjects were being treated with donepezil $5 \mathrm{mg}$ daily, rivastigmine $6 \mathrm{mg}$ daily, or galantamine $16 \mathrm{mg}$ daily at this point. Subjects who had improved continued their current dose. Those who had deteriorated were withdrawn, in keeping with clinical practice. Those who had not improved had the dose increased to $10 \mathrm{mg} /$ day donepezil, or the highest tolerated dose of rivastigmine (usually $4.5 \mathrm{mg}$ twice daily) or galantamine (usually $12 \mathrm{mg}$ twice daily).

\section{Outcome measures}

Response was categorised after 6 months of treatment. To minimise practice effects on test performance, subjects were not tested in the latter 3 months of this period. Subjects were assessed at the same time of day on each visit. At each follow up, the MMSE and DSST were undertaken by researchers blinded to the results of the carer's assessment on the NOSGER. Scores were not fed back to the patient or carer until global outcome had been agreed.

Positive cognitive change was defined as a gain of at least two points over the baseline MMSE score. ${ }^{32}$ Positive behavioural change was defined as an improvement of the combined score on the instrumental activities of daily living (IADL) and social behaviour (SB) subscales of the NOSGER, or maintenance of maximum score. Positive global change was defined as a tripartite agreement amongst doctor, subject, and carer that appreciable improvement had occurred, based upon global impressions. The total number of variables rated as positive ("positive outcome criteria") were calculated for each subject. "Good response" was defined as the presence of at least two out of three positive outcome criteria.

\section{Statistical analysis}

Age, MoM1, MoM2, MMSE, DSST, IADL, and SB scores all had normal distribution (Kolmogorov-Smirnov test). Paired $t$ test analyses assessed differences between MMSE, DSST, IADL, and SB scores at baseline and 6 months, in the cohort as a whole and within the subgroups of "good" or "poor" responders. Independent $t$ test statistics were used to compare these subgroups with each other.

\section{Logistic regression procedure}

Baseline MMSE, DSST, IADL, and SB scores were compared with outcome classification using receiver operational characteristic analysis. Each variable was dichotomised at the point that maximised sensitivity and specificity. Age was dichotomised into groups $\geqslant 75 v<75$ years. MoMl values were dichotomised using the value 0.79 , the suggested cut off point for diagnosing $\mathrm{AD},{ }^{31}$ and MoM2 dichotomised around the age related mean for MoM (1.00). CT appearances of vascular change were dichotomised into "present" or "absent". Non-smokers were defined as those not using cigars or cigarettes for at least 6 months, although in fact, all ex-smokers had not smoked for a much longer period. Variables had their predictive usefulness assessed on the cohort who completed 6 months' treatment. Significant odds ratios (OR) are reported. All analyses were undertaken using SPSS for Windows (version 10.0). 
Table 1 Comparison of good and poor outcome groups after 6 months' treatment

\begin{tabular}{|c|c|c|c|c|c|c|}
\hline \multirow[b]{2}{*}{ Response } & \multicolumn{3}{|l|}{ Baseline } & \multicolumn{3}{|l|}{6 months } \\
\hline & Good & Poor & $p$ & Good & Poor & $\mathbf{p}$ \\
\hline MMSE & $22.56(3.36)(59)$ & $22.59(3.67)(81)$ & $>0.1$ & 24.95 (3.97) (59) & $21.33(4.41)(81)$ & $<0.0005$ \\
\hline IADL & 16.42 (4.69) (59) & $17.20(4.15)(81)$ & $>0.1$ & 18.35 (4.69) (59) & $15.26(4.11)(74)$ & $<0.0005$ \\
\hline SB & $18.53(4.65)(59)$ & $17.60(4.86)(81)$ & $>0.1$ & 20.78 (4.73) (59) & $16.30(5.45)(74)$ & $<0.0005$ \\
\hline DSST & $18.96(12.14)(59)$ & $14.81(11.10)(80)$ & 0.038 & $20.00(13.34)(58)$ & 13.34 (11.07) (78) & 0.002 \\
\hline MoM1 & $0.67(0.16)(48)$ & $0.65(0.18)(64)$ & $>0.1$ & & & \\
\hline MoM2 & 1.17 (0.19) (55) & 1.11 (0.19) (71) & 0.086 & & & \\
\hline AGE & 75.84 (8.37) (59) & 76.61 (7.71) (81) & $>0.1$ & & & \\
\hline
\end{tabular}

\section{RESULTS}

In the study, 160 subjects commenced treatment, but seven could not tolerate treatment for more than 1 week and were not entered into the cohort database. Of the remaining 153, six were withdrawn within 3 months because of intolerance; five were withdrawn after 3 months' treatment because of deterioration in their mental state; one was withdrawn after 3 months because of non-compliance, and one left the catchment area and was lost to follow up. Analysis was thus performed on 140 subjects completing 6 months' treatment, 126 of whom had MoM2 measurement performed.

Mean (SD) age of the cohort (48 men, 92 women) was 76.30 (7.97) years at baseline; $17.1 \%$ were current smokers and $30 \%$ had either current hypertension or were receiving anti-hypertensive treatment. Baseline mean (SD) scores were: MMSE (22.58 (3.53), IADL (16.88 (4.37), SB (17.99 (4.78), and DSST (16.58 (11.70). Mean (SD) scores at 6 month follow up were: MMSE 22.80 (4.63), IADL 16.59 (4.62), SB (17.98 (5.15), and DSST 16.30 (12.46). Although none of the changes was significant at 6 months, there was a significant improvement over baseline in MMSE $(p<0.0005)$ and IADL $(p=0.025)$ after 3 months. The 13 subjects who either did not complete 3 months' treatment or who were withdrawn after 3 months' treatment did not differ significantly on any baseline measure from the cohort completing 6 months' treatment.

\section{Outcome}

Using our criteria, 59 subjects $(42.1 \%)$ of the cohort showed good response after 6 months' treatment (38.6\% of the 153 subjects tolerating treatment for more than 1 week). Of the 81 subjects $(57.9 \%)$ who showed poor response, 17 had lower scores on each of MMSE, IADL, and SB scales than at baseline. By definition, differences in cognition and function between good and poor responders following treatment would be expected. Table 1 illustrates the differences between the groups.

\section{Logistic regression analysis}

Only MoM2 (OR 3.16; 95\% confidence interval (CI) 1.17 to $8.51)$ and DSST $(2.85 ; 1.42$ to 5.73$)$ showed significant odds ratios in the completed cases. Increased age, current smoking, hypertension, and vascular change on CT did not generate significant OR.

\section{Improving prediction}

DSST scores correctly predicted more response categories than MoM2 values in the logistic regression analysis. However, these variables had a different pattern of sensitivity and specificity. A DSST score $\geqslant 18$ had a sensitivity for good response of 0.59 (95\% CI 0.44 to 0.73 ) and a specificity of 0.66 ( $95 \%$ CI 0.53 to 0.77 ). MoM2 values $\geqslant$ the age adjusted median had a sensitivity for good response of 0.89 (95\% CI 0.75 to 0.96 ) and a specificity of 0.30 (95\% CI 0.18 to 0.43 ). Correlation between the MoM2 and DSST scores was only -0.072 ( $\mathrm{n}=126 ; \mathrm{p}>0.1$ Pearson's $\mathrm{r}$, two tailed). Response was therefore tabulated initially using the cut off point for DSST scores, then by cut off point for MoM2. Results are shown in fig 1 . Scores above the cut off point for both variables were associated with a higher likelihood of good response than if one or both were below the cut off point $\left(\chi^{2}=10.61, \mathrm{df}=1, \mathrm{p}=0.001\right)$. Although the absolute increase in prediction of good response increased only to $60.4 \%$ from $42.1 \%$ by undertaking the DSST and MoM2, the difference in the proportion of good responders with high DSST and low atrophy (29/48) and those with one or both below the cut point $(26 / 77)$ was sufficient for the study to

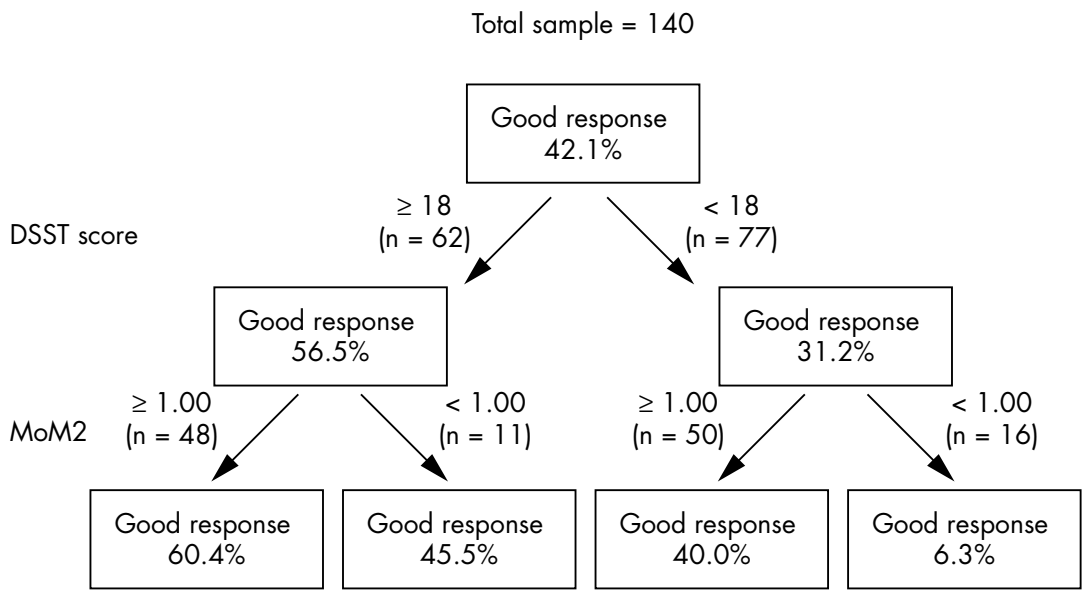

Figure 1 Effect of dichotomising DSST score and MoM2 scores on outcome in subjects completing 6 months treatment. Some subjects did not have MTL thickness measured. 
have greater than $80 \%$ power at this sample size (two tailed $\alpha=0.05)$. $^{33}$

\section{DISCUSSION}

In this study, a combination of baseline performance on the DSST and the degree of medial temporal lobe atrophy appeared to distinguish subjects who had a good response to cholinesterase inhibitors from those with poor response. These variables may differ from those that predict responders to treatment with an active drug from placebo response. The poor response group showed decline in cognition that was similar to untreated patients. ${ }^{34}$ Decline in function of untreated patients does not appear to have been described. Therefore, we cannot comment on the rate of decline in poor responders relative to untreated patients. A $20 \%$ decline in function over 1 year has been described in placebo treated subjects. ${ }^{35}{ }^{36}$ Our data demonstrate the differences in cognition and function between good and poor response that might be expected in a clinical setting. These differences are recognisable to patients and carers, and are clinically significant.

The DSST measures the speed of processing of information, ${ }^{37}$ which is in part dependent upon cholinergic transmission. ${ }^{38}$ Processing speed could therefore be expected to improve in those who respond to cholinesterase inhibitors, and it is encouraging that the DSST has some predictive utility in determining response. Although DSST scores have relatively large standard deviations at any given age range, ${ }^{28}$ the test is rapid and easy to perform. We recommend further assessment of this measure, and exploration of the effect of processing speed on response.

Our measure of MTL atrophy was obtained using an adaptation of CT scanning, which is widely accessible by psychiatrists dealing with the elderly in the UK and is relatively inexpensive. Lesser MTL atrophy was associated with better response.

Although the accuracy of MTL measurement as described by Jobst $e a l^{31}$ has been questioned, ${ }^{39}$ our measure (MoM2) is not of minimum thickness and may be easier to reproduce in other centres than MoMl. Given the variability in reports of atrophy of MTL in $\mathrm{AD}^{31}{ }^{40}$ we support the view that standardisation of MTL measurement is required.

The MMSE, although widely used in drug studies, is not designed for that purpose. This study shows that the MMSE at baseline is not a good predictor of response. However, the number of patients whose MMSE improved by at least one point after 6 months $(53.7 \%)$ is substantially greater than that reported in a longitudinal study of untreated patients, ${ }^{41}$ suggesting that the improvement is unlikely to be due to the natural history of AD.

Potential risk factors such as older age, hypertension, and current smoking did not generate a significant OR in our study. The number of current smokers was low, but similar to the Scottish prevalence in this age group. We did not assess the influence of vascular risk on response, ${ }^{8}$ but instead assessed significance of vascular change using a combination of neuroradiographic appearance and clinical features. Although we recognise that CT scanning is less sensitive than MRI in detecting vascular lesions, ${ }^{42}$ lesions seen on CT have proved more accurate at predicting the development of neurological signs ${ }^{42}$ and are associated with neurological symptoms. ${ }^{27}$ The OR associated with vascular change that is not considered clinically significant using our definition would suggest that vascular change on CT scan per se is not associated with an altered rate of response.

Using a very restrictive cut off point for vascular risk factors on the Rosen scale ${ }^{22}$ and excluding subjects meeting our definition of clinically significant vascular disease, we believe that our population represents $\mathrm{AD}$ that is not coloured by vascular pathology. It is disappointing, therefore, that response rates to cholinesterase inhibitors were not better than we found, even though dropout rates in our clinical setting were much lower than expected based on published trials. The characteristics of our population, pattern of MMSE response, ${ }^{26}$ and response rate are similar to those reported in clinical trials ${ }^{1-3}$ and UK memory clinics. ${ }^{6}{ }^{43}$ Therefore, it may be possible to generalise the results of this study to a wider population.

Ideally, the usefulness of our measure of MTL thickness requires assessment in other centres, and a fully prospective trial comparing the actual outcome of patients with none, one, or two of our suggested predictive factors using raters blinded to baseline classification would be justified by our study. Such a trial would reduce the problems associated with the analysis of multiple variables. Until then, given the current sensitivity and specificity of our measures, we would advise caution in using the DSST and MoM2 as the only measures to determine whether treatment is indicated for an individual patient. The combination of the DSST and MoM2 could be argued to have a relatively small effect compared to the use of no measures. However, a response rate of $60 \%$ is similar to that for antipsychotics or antidepressants, and may encourage prescription of cholinesterase inhibitors. We recognise that our measures appear to be a better predictor of poor than good response. Because the number of subjects with scores below the cut off point of both the DSST and MoM2 is small, caution must be used in restricting treatment to this group, despite only one of our subjects in this category showing good response at 6 months.

We have not assessed the usefulness of our predictive items in subjects with other types of dementia and cannot recommend their use as predictors of response to cholinesterase inhibitors in the absence of a clear diagnosis of $\mathrm{AD}$. We hope that our study, in a clinical setting, stimulates the seeking of clinical or radiological parameters that might be more predictive of good response. The identification of these parameters is now vital if the benefits of cholinesterase inhibitors are to be maximised.

\section{ACKNOWLEDGEMENTS}

We acknowledge the contribution of Professor I C Reid, Department of Psychiatry, University of Aberdeen, who provided critical review of previous drafts of this paper and supervised the research work. Dr R C Chen, Senior Lecturer, Department of Epidemiology and Public Health, University of Dundee provided statistical advice. Our colleague Dr G Cousland contributed to the data collection. Invaluable secretarial support was provided by $\mathrm{H}$ Neil.

\section{Authors' affiliations}

P J Connelly, Department of Psychiatry, University of Dundee, Murray Royal Hospital, Muirhall Road, Perth, PH2 7BH, UK

N P Prentice, Senior Lecturer in Old Age Psychiatry, Department of Psychiatry, University of Dundee, Murray Royal Hospital, Muirhall Road, Perth, PH2 7BH, UK

K G Fowler, Consultant Radiologist, Perth Royal Infirmary, Taymount Terrace, Perth PHI 1NX, UK

There was no funding source or sponsor for this work.

Competing interests: P J Connelly and N P Prentice have received honoraria and travel sponsorship from manufacturers of currently licensed cholinesterase inhibitors. P J Connelly and N P Prentice have received research funds from Eisai/Pfizer. K G Fowler has no competing interests.

\section{REFERENCES}

1 Burns A, Rossor M, Hecker J, et al. The effects of donepezil in Alzheimer's disease-results from a multinational trial. Dement Geriatr Cogn Disord 1999; 10:237-44.

2 Rösler M, Anand R, Cicin-Sain A, et al. Efficacy and safety of Rivastigmine in patients with Alzheimer's Disease: international randomised controlled trial. BMJ 1999;318:633-40. 
3 Tariot PN, Solomon PR, Morris JC, et al. A 5-month, randomized, placebocontrolled trial of galantamine in AD. The Galantamine USA-10 Study Group. Neurology 2000;54:2269-76

4 Lilienfeld S, Parys W. Galantamine: additional benefits to patients with Alzheimer's Disease. Dement Geriatr Cogn Disord 2000;11(suppl 1):19-27.

5 Farlow MR, Hake A, Messina J, et al. Response of patients with Alzheimer disease to rivastigmine treatment is predicted by the rate of disease progression. Arch Neurol 2001;58:417-22.

6 Evans $M$, Ellis A, Watson D, et al. Sustained cognitive improvement following treatment of Alzheimer's disease with donepezil. Int J Geriatr Psychiatry 2000;15:50-3

7 Farlow MR, Schneider LS. Rivastigmine and response to treatment in different subgroups of patients with Alzheimer's Disease. NeurobiolAging 1998;(suppl 4S): 1264.

8 Kumar V, Anand R, Messina J, et al. An efficacy and safety analysis of Exelon in Alzheimer's disease patients with concurrent vascular risk factors. Eur J Neurol 2000;7:159-69.

9 Poirier J, Delisle M-C, Quirion R, et al. Apolipoprotein E4 allele as a predictor of cholinergic deficits and treatment outcome in Alzheimer's Disease. Proc Natl Acad Sci USA 1995:92:12260-4

10 Aerssens J, Raemaekers P, Lilienfield S, et al. APOE genotype: no influence on galantamine treatment efficacy nor on rate of decline in Alzheimer's disease. Dement Geriatr Cogn Disord 2001; 12:69-77.

11 Lucotte G, Oddoze C, Michel B-F. Apolipoprotein E genotype allele epsilon4 and response to tacrine in Alzheimer's disease. Alzheimer's Res 1996;2:101-2.

12 Riekkinen P Jr, Soininen H, Helkala EL, et al. Hippocampal atrophy, acute THA treatment and memory in Alzheimer's disease. Neuroreport 1995:6:1297-300.

13 Riekkinen P Riekkinen Jr M, Soininen $\mathrm{H}$, et al. Frontal dysfunction blocks the therapeutic effect of THA on attention in Alzheimer's disease. Neuroreport 1997;8:1845-9.

14 Tanaka $Y$, Hanyu $\mathrm{H}$, Sakurai $\mathrm{H}$, et al. Atrophy of the substantia innominata on magnetic resonance imaging predicts response to donepezil treatment in Alzheimer's disease patients. Dement Geriatr Cogn Disord 2003;16:1 19-25

15 Folstein MF, Folstein SE, McHugh PA. Mini Mental State: A practical method for grading the cognitive state of patients for the clinician. J Psychiatr Res 1975; 12:189-98.

16 Sahakian BJ, Owen AM, Morant NJ, et al. Further analysis of the cognitive effects of tetrahydroaminoacridine (THA) in Alzheimer's disease: assessmen of attentional and mnemonic function using CANTAB. Psychopharmacology 1993; 110:395-401.

17 Alhainen K, Helkala EL, Riekkinen P. Psychometric discrimination of tetrahydroaminoacridine responders in Alzheimer patients. Dementia 1993;4:54-8

18 Almkvist O, Jelic V, Amberla K, et al. Responder characteristics to a single oral dose of cholinesterase inhibitor: a double-blind placebo-controlled study with tacrine in Alzheimer patients. Dement Geriatr Cogn Disord 2001; 12:22-30.

19 Knott V, Mohr E, Mahoney C, et al. Pharmaco-EEG test dose response predicts cholinesterase inhibitor treatment outcome in Alzheimer's Disease. Methods Find Exp Clin Pharmacol 2000;22:115-22.

20 Adler G, Brassen S, Chwalek K, et al. Prediction of treatment response to rivastigmine in Alzheimer's dementia. I Neurol Neurosurg Psychiatry 2004;75:292-4.

21 McKhann G, Drachman D, Folstein M, et al. Clinical diagnosis of Alzheimer's disease: report of the NINCDS-ADRDA work group under the auspices of the Department of Health and Human Task Force in Alzheimer's disease. Neurology 1984;34:939-44
22 Rosen WG Terry RD, Fuld PA, et al. Pathological verification of ischaemic score in differentiation of dementias. Ann Neurol 1980;7:486-8

23 Tarvonen-Schröeder S, Röyttä M, Räihä I, et al. Clinical features of leukoaraiosis. J Neurol Neurosurg Psychiatr 1996;60:431-6.

24 Villardita C, Grioli S, Lomeo C, et al. Clinical studies with oxiracetam in patients with dementia of Alzheimer type and multi-infarct dementia of mild to moderate degree. Neuropsychobiology 1992;25:24-8.

25 Gupta SR, Naheedy MH, Young JC, et al. Periventricular white matter changes and dementia. Clinical, neuropsychological, radiological, and pathological correlation. Arch Neurol 1988:45:637-41.

26 Almkvist O, Wahlund LO, Lundman G, et al. White matter hyperintensity and neuropsychological functions in dementia and healthy ageing. Arch Neurol 1992;49:626-32.

27 McDonald C. Clinical heterogeneity in senile dementia. Br J Psychiatry 1969:115:267-71.

28 Wechsler D. Wechsler Adult Intelligence Scale-Revised. New York: The Psychological Corporation, 1981

29 Luria AR. Higher cortical functions in man. London: Tavistock, 1966.

30 Spiegel R, Brunner C, Ermini-Funfschilling D, et al. A new behavioural assessment scale for geriatric out- and in-patients: the NOSGER (Nurses Observation Scale for Geriatric Patients). J Am Geriatr Soc 1991;39:339-47.

31 Jobst KA, Smith AD, Szatmari M, et al. Detection in life of confirmed Alzheimer's Disease using a simple measurement of medical temporal lobe atrophy by compacted tomography. Lancet 1992;340:1183-8.

32 Iverson GL. Interpretation of Mini-Mental State Examination scores in community-dwelling elderly and geriatric neuro-psychiatry patients. Int J Geriatr Psychiatry 1998;13:661-6.

33 Cohen J. Statistical power analysis for the behavioural sciences, 2nd ed. Hillsdale, New Jersey: Lawrence Erlbaum Associates, 1988.

34 Han L, Cole M, Bellavance F, et al. Tracking cognitive decline in Alzheimer's disease using the mini-mental state examination: a meta-analysis. Inl Psychogeriatr 2000;12:231-47.

35 Raskind MA, Peskind MD, Wessel T, et al. Galantamine in AD: A six month randomized placebo controlled trial with a six month extension. Neurology 2000;54:2261-8

36 Winblad B, Engedal K, Soininen $\mathrm{H}$, et al. A one year randomized placebocontrolled study of donepezil in patients with mild to moderate AD. Neurology 2001;57:489-95.

37 Salthouse TA. What do adult age differences in the Digit Symbol Substitution Test reflect? J Gerontol 1992;47:121-128.

38 Muir JL, Everitt BJ, Robbins TW. Reversal of visual attentional dysfunction following lesions of the basal forebrain by physostigmine but not by the 5HT3 receptor antagonist ondansetron. Psychopharmacology 1995;1 18:82-92.

39 Oksengaard AR, Haakonsen M, Dullerud R, et al. Accuracy of CT scan measurements of the medial temporal lobe in routine dementia diagnostics. Int J Geriatr Psychiatry 2003;18:308-12.

40 Luce A, McKeith I, Swann A, et al. How do memory clinics compare with traditional old age psychiatry services. Int J Geriatr Psychiatry $2001 ; 16: 837-45$

41 Holmes C, Lovestone S. Long-term cognitive and functional decline in lateonset Alzheimer's disease: therapeutic implications. Age Ageing 2003;32:200-4.

42 Lopez OL, Becker JT, Jungreis CA, et al. Computed tomography but not magnetic resonance imaging identified periventricular white-matter lesions predict symptomatic cerebrovascular disease in probable Alzheimer's disease. Arch Neurol 1995:52:659-64.

43 Matthews HP, Korbey J, Wilkinson DG, et al. Donepezil in Alzheimer's disease: eighteen month results from Southampton Memory Clinic. Int J Geriatr Psychiatry 2000;15:713-20. 